\title{
Left ventricular aneurysm in the fetus: a diagnosis with a mixed prognosis
}

\author{
Doff B. McElhinney, Norman H. Silverman \\ Division of Pediatric Cardiology, University of California, San Francisco, USA
}

$I^{2}$ N THIS ISSUE OF Cardiology in the Young, Matias and colleagues ${ }^{1}$ describe 3 cases of left ventricular aneurysm detected by prenatal echocardiography. Prior to this series, a mere 9 cases had been published. ${ }^{2-7}$ As such, this report must be considered a substantial addition to the literature on this topic. At the same time as the cases described by Matias et al. ${ }^{1}$ can be said to further our knowledge of this rare condition, however, they also serve to confound our understanding of it. Insofar as their outcome was uniformly poor, the ultimate fate of these 3 fetuses differed substantially from the majority of the previously reported experience. Of the 9 previously described cases, 6 survived to birth and were asymptomatic postnatally, 2 aborted spontaneously, and 1 pregnancy was terminated after the identification of fetal hydrops. By opening our eyes more fully to the breadth and balance of the prognostic spectrum for this condition, this report simultaneously compels us to acknowledge how little we know about it. What are the causes? What is the natural history? Perhaps most important, are there historical, morphologic, or physiologic factors that can help us predict which fetuses will do well and which will do poorly?

The value of this report lies not simply in its addition of 3 new cases to the existing literature, and its challenge to us to strive for improved understanding of fetal left ventricular aneurysm, but more so in the unprecedented extent to which the physiology of this condition is detailed. Matias et al. provide us with important new information about the potential functional disturbances that

Correspondence to: Doff B. McElhinney, MD, Children's Hospital of Philadelphia, 34th Street and Civic Center Blvd, Rm 9557, Philadelphia, PA 19104, USA. Tel: (215)590-1000; Fax: (215)590-2768 can be engendered by a large aneurysm of the fetal left ventricle, as well as a glimpse at the physiologic factors that are likely to determine outcome. Their report confirms the findings of CavalléGarrido and colleagues, ${ }^{7}$ namely, that left ventricular aneurysms in the fetus can enlarge, just as we know they can diminish in relative size, and suggests that such enlargement may be an indication of poor prognosis. The authors also document perturbed ventricular mechanics, dysfunction of the mitral valve, and consequent left-to-right shunting at atrial level. This latter finding indicates significant impairment of left heart function, and has been associated with unfavorable prognosis in fetuses with various forms of structural heart disease. ${ }^{8}$ It is not surprising to learn that it may also be associated with poor outcome of left ventricular aneurysm in the fetus.

Reviewing the report of Matias and colleagues, ${ }^{1}$ along with the 9 previously described cases of fetal left ventricular aneurysm and 2 that have been diagnosed at our institution since 1993 (see Table 1 and Fig. 1), we have discerned several prognostic patterns. Among these 14 patients, 7 were delivered and survived with no symptoms. Of the other 7,3 miscarried, 3 were aborted after identification of effusions or hydrops, and 1 died in the neonatal period. In 6 of the 7 fetuses with poor outcome, the aneurysm was detected at 24 weeks estimated gestational age or earlier, whereas diagnosis of 5 of 7 survivors occurred in the third trimester (Fig. 2). In addition, 5 of the 7 fetuses with a poor outcome had a ratio of volumes between aneurysm and left ventricle of 1 or greater. This measure was not specified for the other 2, 1 of which is indicated in Fig. 2 as having a ratio of greater than unity, which appears to be the case from the figures included in the report of Sepulveda and colleagues.' Among fetuses that 


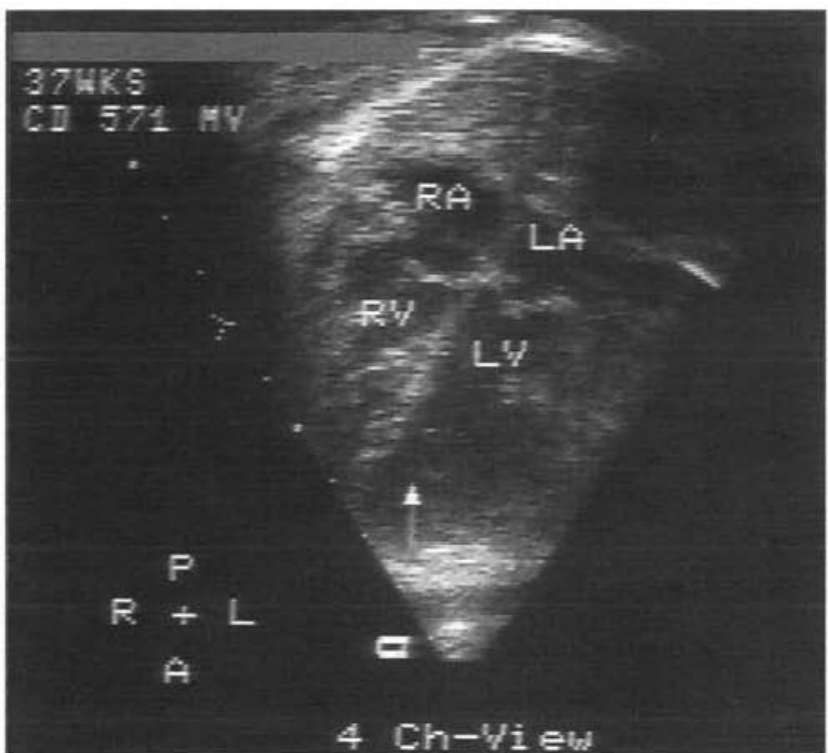

(a)

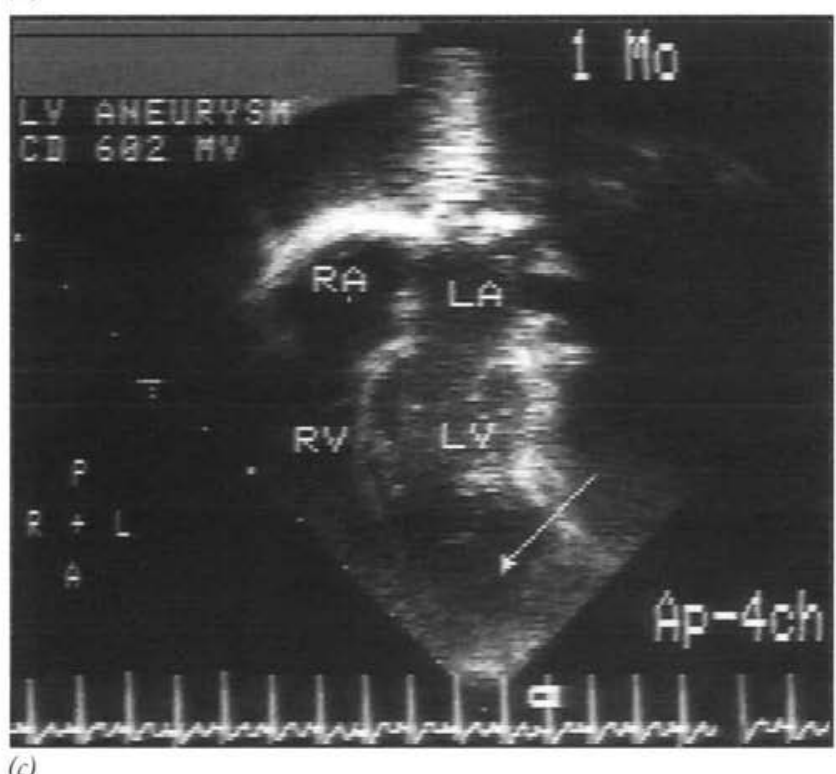

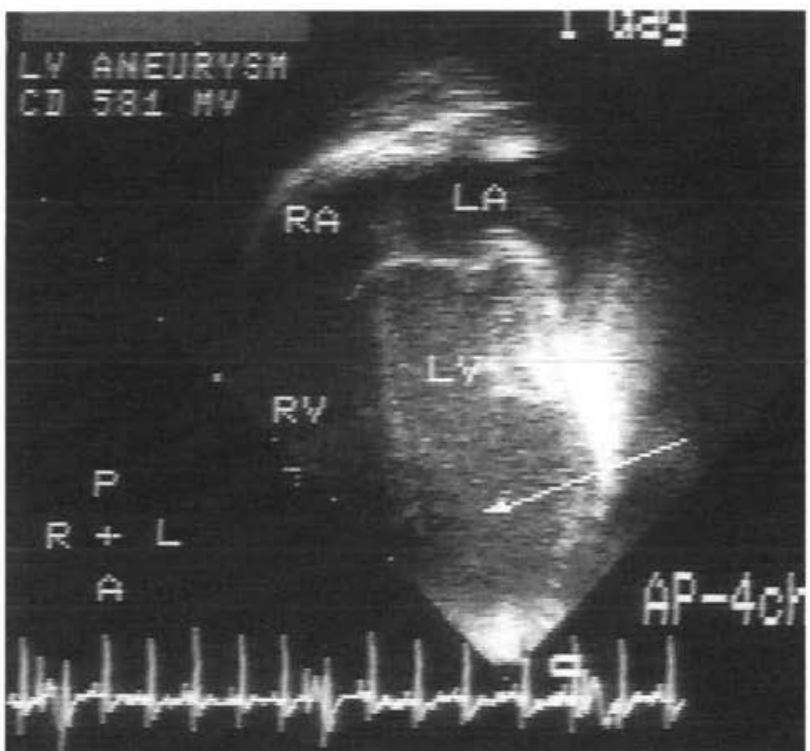

(b)

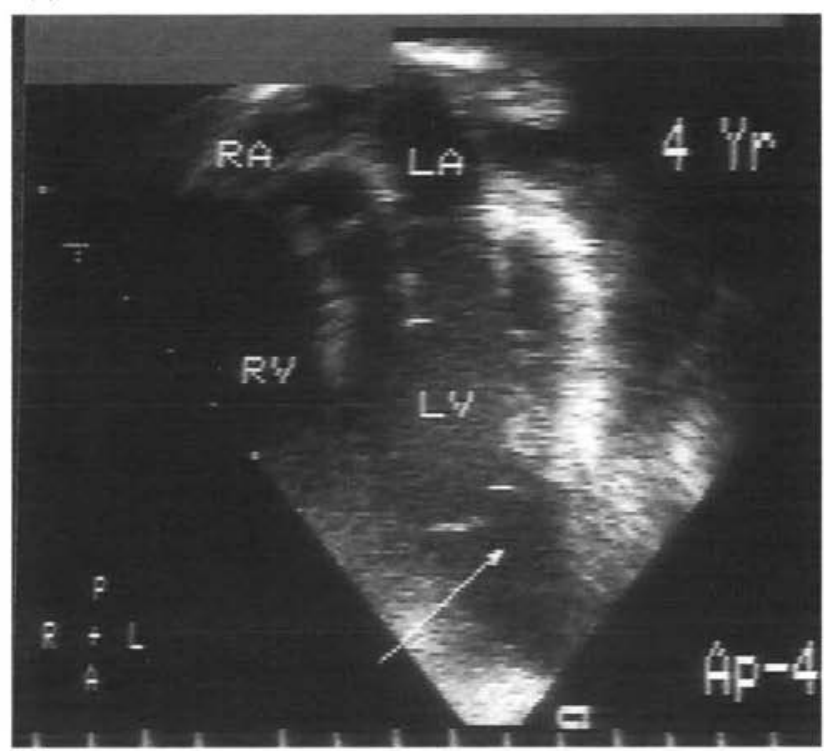

(d)

Figure 1.

Prenatal and postnatal echocardiograms from the 4-chamber view in a patient of ours diagnosed with an apical left ventricular aneurysm at 37 weeks of fetal life. A) At the time of fetal diagnosis, the aneurysm to left ventricular volume ratio was approximately 0.7. The size and function of the ventricles and atrioventricular valves were within normal limits, and there was no evidence of fetal bydrops. Two weeks after diagnosis, the mother delivered a bealtby female infant who bad no of cardiovascular or respiratory symptoms. B) An echocardiogram on the first day of life confirmed a wide-mouthed aneurysm of the left ventricular apex, with no significant compromise of left ventricular or mitral valvar function. Echocardiograms were repeated at C) 1 month, 1 year, 2 years, and D) 4 years of age, and continued to show these findings. As in 1 of the cases reported by Matias et al. ${ }^{1}$ and that described by Gembruch and colleagues ${ }^{2}$, there appeared to be thin, membranous strands of tissue extending from the ventricular wall at the junction of mouth of the aneurysm, which can be seen most clearly in D). At the 4-year echocardiogram, the ratio of volume of the aneurysm to left ventricular volume was only 0.4 .

survived, all had a ratio that either was documented to be less than unity, or else was not specified.

As Matias et al. allude to in their discussion, it is intuitive that a larger aneurysm should correspond with poor outcome, as it may place a significant volume load on the fetal heart, which has a limited functional reserve. Secondary changes resulting from this process may lead to further impairment of cardiac performance, to fetal hydrops, and possibly to pulmonary hypoplasia, which Matias et al., ${ }^{1}$ for the first time, have documented in association with fetal ventricular aneurysm. 
Table 1. Details of 2 fetuses diagnosed with left ventricular aneurysm since 1993 at UCSF

\begin{tabular}{lll}
\hline & Fetus 1 & Fetus 2 \\
\hline Gestational age (wks) & 37 & 22 \\
LVA:LV volume ratio & 0.7 & 1.5 \\
Location of LVA & Apex & Apex \\
LV function & Normal & Diastolic \& systolic function depressed, to and fro flow \\
& Normal & into LVA \\
Mitral valvar function & Normal \\
Flow across oval foramen & Right to left & Minimal, bidirectional \\
Fetal hydrops & No & Mild \\
Outcome & Delivered at 39 wks, alive and & Pregnancy terminated at 23 wks \\
& asymptomatic 5 yrs later & \\
\hline
\end{tabular}

LV: left ventricle; LVA: left ventricular aneurysm

The apparent fact that larger aneurysms have a poorer prognosis begs the question, what factors determine the size of an aneurysm? As the authors discuss, ${ }^{1}$ the etiology of this condition is not known. Prenatally diagnosed ventricular aneurysms probably result from the same process(es) as give rise to those identified after birth, for which vascular and infectious causes are the leading candidates. They may, in fact, be one and the same phenomenon, distinguished only by the time point of diagnosis. In such a scenario, the initial size of the aneurysm is likely to be a function of the extent of the insult. Those that are larger initially may be susceptible to subsequent evolution, perhaps as a result of elevated mural stress in the aneurysmal segment. The size of the aneurysm may also correlate with the point in gestation at which it develops. All but 1 of the fetuses with a ratio of volumes greater than unity were diagnosed at 24-weeks gestation or earlier. Of course, there is no way of knowing when aneurysms diagnosed in the third trimester initially developed, nor their original size. Although this uncertainty may confound efforts to deduce factors of prognostic significance, it is reassuring that Cavallé-Garrido and colleagues ${ }^{7}$ documented relative diminution or stability on serial echocardiograms of aneurysms diagnosed in second trimester fetuses with initial ratios of volumes less than 1 .

Clearly, left ventricular aneurysm in the fetus is a diagnosis with a mixed prognosis. In daily practice, pediatric cardiologists will encounter this problem rarely, but when they do it will be important to have a basis for predicting where along the prognostic spectrum a particular case will fall. This has significant implications for counselling the pregnant mother. As discussed above, there are several diagnostic factors that appear to be helpful in this respect. Of 8 fetuses diagnosed at or before 24 weeks gestation, 6 had a poor outcome. Five of those had a ratio of volumes equal to or greater

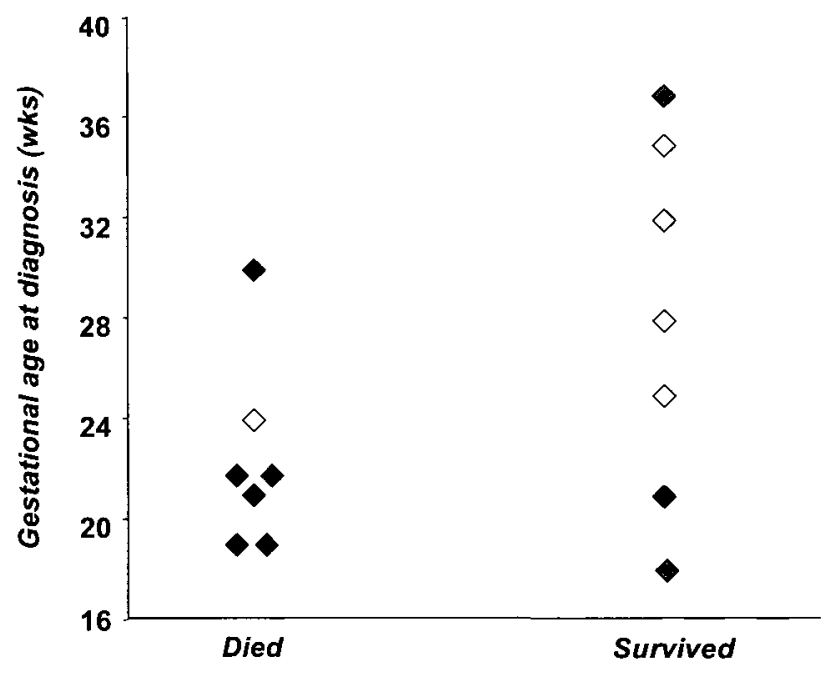

Figure 2.

As demonstrated in this figure, which includes all previously reported cases as well as those described by Matias et al. \{1\} and in this editorial, fetuses diagnosed with left ventricular aneurysm at 24 weeks estimated gestational age or earlier are less likely to survive than those diagnosed in the third trimester. In addition, the size of the aneurysm relative to the size of the left ventricle appears to be an important factor in predicting survival, as described in the text. The shading of the symbols indicates the ratio of volumes of aneurysm to left ventricle, with solid diamonds representing fetuses with a ratio of 1 or greater (includes 1 fetus reported by Matias et al. with a ratio of 0.98 ), batched diamonds representing a ratio less than unity, and bollow diamonds representing cases in which the size of the aneurysm and/or left ventricle were not specified.

than unity. The 2 fetuses that survived after diagnosis in the second trimester both had ratios documented to be less than 1 . Unfortunately, detailed physiologic evaluation was not provided in the 9 previously reported cases. Nevertheless, it seems reasonable to propose that hydrops or effusions, enlargement of the aneurysm as seen on serial echocardiograms, significant mitral regurgitation, poor left ventricular function, and a left-to-right 
atrial shunt, all herald a poor outcome. Although the available data are too limited to perform meaningful statistical analysis, it seems likely that, in many cases, earlier age at diagnosis, larger size of the aneurysm, and the foregoing physiological factors all point toward poor outcome. These findings will probably cluster together, as many are interrelated. In addition, despite the detailed functional information provided by Matias et al., ${ }^{1}$ there remain several potentially important physiologic factors that require characterization, including patterns of systemic and umbilical vascular flows, and functional integrity of the right heart.

Fetal echocardiography is a well-established diagnostic practice that may improve the management of fetuses and neonates with heart disease in a number of ways. By contributing to our understanding of the natural history of complex congenital heart disease during fetal life, prenatal echocardiography allows for more informed consideration of the options after the diagnosis of an affected fetus. The ability to diagnose heart disease increasingly early in gestation may facilitate decision-making regarding termination of pregnancy, ${ }^{9}$, transport to a neonatal cardiac surgery center for delivery and surgery, which may have a salutary effect on outcomes, ${ }^{10}$ and perhaps someday prenatal cardiac surgery. ${ }^{11}$ With respect to aneurysms of the fetal left ventricle, the limited findings documented to date conform to a relatively encouraging paradigm for the role of fetal echocardiography. That is, the estimated volume of the aneurysm relative to that of the left ventricle appears to correspond with prognosis, particularly in fetuses diagnosed before the third trimester. In some respects, it is this group of fetuses in which accurate and informed prognostication is of most critical import. At this stage, mothers still have the option of terminating the pregnancy in most countries where fetal echocardiography is performed, which may heighten the potential implications of accurate or inaccurate prognosis. We anticipate that further characterization of physiologic parameters will enhance the sensitivity and specificity of the aforementioned criterions.
Matias and colleagues ${ }^{1}$ are to be commended for a thorough evaluation of fetal left ventricular aneurysm, for bringing our attention to the paucity of information that exists about this lesion, and for helping to demonstrate the potential clinical utility of fetal echocardiography. Of course, there remains much to be learned about this unusual condition, but the report by Matias et al. should be acknowledged for significantly advancing the level of our knowledge.

\section{References}

1. Matias A, Fredouille C, Nesmann, Azancot A. Prenatal diagnosis of left ventricular aneurysm: a report of three cases and a review. Cardiol Young 1999;9(2): 163-172

2. Gembruch U, Steil E, Redel DA, Hansmann M. Prenatal diagnosis of a left ventricular aneurysm. Prenat Diagn 1990;10 203-209.

3. Jacobson RL, Perez A, Meyer RA, Miodovnik M, Siddiqi TA Prenatal diagnosis of fetal left ventricular aneurysm; a case report and review. Obstet Gynecol 1991;78: 525-527.

4. Hornberger LK, Dalvi B, Benacerraf BR. Prenatal sonographic detection of cardiac aneurysms and diverticula. J Ultrasound Med 1994;967-970

5. Sepulveda W, Drysdale K, Kyle PM, McNeal AD, Moore IE Congenital left ventricular aneurysm causing hydrops fetalis: prenatal diagnosis with color Doppler ultrasonography. J Ultrasound Med 1996;15: 327-331.

6. Sherman SJ, Leenhouts KH, Utter GO, Litaker M, Lawson P. Prenatal diagnosis of left ventricular aneurysm in the late second trimester: a case report. Ultrasound Obstet Gynecol 1996;7: 456-457.

7. Cavallé-Garrido T, Cloutier A, Harder J, Boutin C, Smallhorn $\mathrm{JF}$. Evolution of fetal ventricular aneurysms and diverticula of the heart: an echocardiographic study. Am J Perinatol 1997; 14: 393-400.

8. Berning RA, Silverman NH, Villegas M, Sahn DJ, Martin GR, Rice MJ. Reversed shunting across the ductus arteriosus or atrial septum in utero heralds severe congenital heart disease. J Am Coll Cardiol 1996;27;481-486.

9. Allan LD. Fetal diagnosis of congenital heart disease. Cardiol Young 1996;6: 258

10. Tworetzky W, McElhinney DB, Reddy VM, Hanley FL, Silverman NH. Does prenatal diagnosis of hypoplastic left heart syndrome improve surgical outcome? J Am Coll Cardiol 1998;31: 71A.

11. Reddy VM, McElhinney DB. Update on prospects for fetal cardiovascular surgery. Curr Opin Pediatr 1997;9: 530-535. 